\title{
Female pelvic actinomycosis and intrauterine contraceptive devices
}

This article was published in the following Dove Press journal:

Open Access Journal of Contraception

10 May 2010

Number of times this article has been viewed

\author{
Faustino R Pérez-López ${ }^{1,2}$ \\ José J Tobajas ${ }^{1,3}$ \\ Peter Chedraui ${ }^{4}$ \\ 'Department of Obstetrics and \\ Gynecology, Facultad de Medicina, \\ Universidad de Zaragoza; ${ }^{2}$ Hospital \\ Clínico Lozano Blesa; ${ }^{3}$ Hospital \\ Universitario Miguel Servet, Zaragoza, \\ Spain; ${ }^{4}$ Enrique C. Sotomayor \\ Obstetrics and Gynecology Hospital, \\ Guayaquil, Ecuador
}

\begin{abstract}
Female genital Actinomyces infection is relatively rare, although strongly related to long-lasting intrauterine contraceptive device (IUD) application. An infective pathway has been postulated extending upward from the female perineum to the vagina and cervix. The traumatic effect of the device and a prior infection may contribute to the Actinomyces infection in the female genitalia. This disease is characterized by local swelling, suppuration, abscess formation, tissue fibrosis, tubal-ovarian mass and fistula formation. The infection spreads by contiguity often mimicking the characteristics of a malignant neoplastic process. Currently there is no consensus regarding diagnosis and screening tests, although there seems to be agreement in relation to IUD type, duration, and sexual behavior as major risk factors.
\end{abstract}

Keywords: contraception, intrauterine contraceptive device, pelvic actinomycosis, sexuality

\section{Introduction}

Actinomyces (A.) are Gram-positive, nonspore anerobic or microaerophilic species that produce chronic course infections, and favor abscess formation, tissue fibrosis and sinus tract in different anatomical locations. There is no person-to-person Actinomyces transmission. These micro-organisms require breaking the mucous membrane integrity and the existence of devitalized tissue to invade deeper body structures. Actinomycosis is usually associated to 8-10 other bacterial types which contribute by elaborating toxins, enzymes and/or by inhibiting host defenses. ${ }^{1}$ Thus, these copathogens increase the low invasiveness of Actinomyces, and may cause treatment failure. After local establishing of actinomycosis, a slowly progressive hematogenous spread is possible. ${ }^{2}$ Most frequently affected locations include: cervicofacial (55\%), thoracic $(20 \%)$ and the pelvic region (15\%). The diseases low prevalence and its different anatomical locations impose a diagnostic challenge to the various medical specialties. ${ }^{3-5}$ The genus Actinomyces includes $A$. israelii, $A$. naeslundii, $A$. viscosus, $A$. meyeri, $A$. odontolyticus and $A$. gerencseriae. A. israelii is the most common subtype in humans and is even considered as a common occasional commensal of the oropharynx, the gastrointestinal tract, and the vagina. ${ }^{6-12}$

Intrauterine contraceptive device (IUD) users may have a high prevalence of genital A. israelii and other Actinomyces types, which can be pathogenic or commensal under different circumstances. ${ }^{1,4,13-15}$ This document will update information regarding the role of Actinomyces in female genital infections in IUD users.
Correspondence: FR Pérez-López Hospital Clínico de Zaragoza, Domingo Miral s/n, Zaragoza 50009, Spain

Tel +34976 76 I734

Fax +34976761735

Email faustino.perez@unizar.es submit your manuscript | www.dovepress.com

Dovepress

7476 


\section{Genital actinomycosis and intrauterine contraceptive devices}

Pelvic actinomycosis is a chronic suppurative and granulomatous infection similar to other forms of pelvic inflammatory disease. However, IUD users with pelvic inflammatory symptoms should be suspected to have an active Actinomyces infection. Reports indicate that $A$. israelii may infect $1.6 \%-11.6 \%$ of IUD users worldwide. ${ }^{4,8,16}$ These figures are mostly related to plastic IUDs without metal or hormonal load. ${ }^{16,17}$ At present there are no identified risk factors that could help screen which women who use IUDs are prone to develop genital or pelvic Actinomyces infection. Despite this speculations have been raised regarding dental caries, oral hygiene, and immune suppression.

Genital actinomycosis has an insidious clinical course, frequently associated to vague lower abdominal pain, fever and bleeding between menstrual periods. Treatment delay favors a torpid evolution and progressive infectious spreading. Symptoms and signs of genital actinomycosis are not specific; thus, a high degree of suspicion is necessary for its diagnosis. Many cases are diagnosed when infection is advanced, when masses or abscesses are found in the pelvis. In the majority of cases, women had used an IUD for a prolonged time and may complain of abdominal pain, weight loss, vaginal discharge, and fever. Early antibiotic treatment may produce complete resolution. Advanced pelvic actinomycosis may mimic genital malignancy, and be frequently submitted to surgery; however, pathological examination has demonstrated its infective nature.

Some classical studies have documented genital Actinomyces (A. israelii) almost exclusively in women IUD or vaginal pessary users. ${ }^{6-9}$ However, $A$. naeslundii, A. viscosus, and $A$. eriksonii may be demonstrated in a similar context. ${ }^{9}$ IUDs induce mild inflammatory changes in the endometrium with necrosis that creates an anerobic environment that favors growth of $A$. israelii and other anerobics. ${ }^{18}$ It seems that plastic IUDs were more likely colonized by Actinomyces than metal-containing devices. In addition, long term use ( $>5$ years) is also a risk factor for the infection.

\section{Diagnosis}

Many women present with pelvic inflammatory symptoms, and positive Papanicolau (PAP) stained cervicovaginal smears have been found adequate for diagnosis. ${ }^{19}$ The presence of Actinomyces-like organisms, A. israelii and other Actinomyces in cervical smears indicate the risk of developing actinomycosis. ${ }^{20}$ In a series of 99,405 cervicovaginal cytologies performed in an 8-year period,
119 IUD users presented with vaginal Actinomyces infection $(0.11 \%)$, including mixed infections $(15.3 \%)$, yeast infection (6.3\%), and bacterial vaginosis $(6.3 \%) .{ }^{21}$ However, due to the low positive predictive value this finding is considered to lack prognostic significance. Thus, the presence of Actinomyces in the PAP smear in the absence of clinical symptoms is no indication for antimicrobial treatment or IUD extraction. ${ }^{4} \mathrm{An}$ insidious clinical course, mild pain, dyspareunia, spotting and pelvic discomfort may be suspicious of endometrial infection associated or not to adnexal infection.

Material obtained from the uterine cavity, fimbrial lumen or pelvis provides the best source for confirming etiological Actinomyces infection. Diagnosis by traditional culture techniques is considered insensitive as Actinomyces is oxygen-sensitive and slow-growing, when compared to concomitant faster-growing anerobes. Actinomyces may culture in an anaerobic transport device. Despite this culture is difficult and definitive identification may require 2-3 weeks. Immunofluorescence technique with direct smears may be a faster diagnostic method. Using a Gramstained smear it is possible to demonstrate beaded, branched, positive stained filamentous rods. Micro-organism diagnosis may be obtained by microscopic examination and the presence of sulphur granules stained with a $1 \%$ methylene blue solution. ${ }^{1}$ Antibiograms are not needed due to known sensitivity to specific antibiotics.

Anemia and mild leukocytosis may be found in cell blood count, and erythrocyte sedimentation rate and $C$-reactive protein found elevated. Serum tumor marker CA 125 is mildly elevated, while $\beta$-chorionic gonadotropin and $\alpha$-fetoprotein are normal, which may be useful to rule out some malignant conditions. Ultrasound exploration may document the pelvic infection when the process is advanced with purulent material in tubes or pelvis. Severe clinical cases and radiological imaging (computed tomography scan) may mimic a malignant pathology and very often lead to complex surgery. Compared to ultrasound, CT-scan can provide high resolution diagnostic imaging, ${ }^{22,23}$ useful for confirming the nonmalignant nature of the disease and avoiding unnecessary risky surgery. In selected pelvic inflammatory cases, laparoscopy is a useful tool for diagnosing pelvic $A$. israelii infection.

In many cases, diagnosis is usually made retrospectively by means of histologic examination of surgically obtained specimens during laparotomy or laparoscopy, but rarely preoperatively. ${ }^{23}$ Tissue histologic studies show suppurative and granulomatous inflammatory changes, connective proliferation, and sulphur granules which have also been 
found in infections caused by Nocardia brasiliensis, Streptomyces madurae, and Staphylococcus aureus. These granules are yellowish particles when seen by the naked eye and are formed by clumps of filamentous Actinomyces surrounded by neutrophils.

\section{Management}

Differential diagnosis of pelvic Actinomyces infection should include malignant genital tumors, pelvic inflammatory disease due to other causes, and endometriosis. Ultrasound and radiological explorations may allow performing biopsies and sampling which may aid in diagnosis. At present no procedure is capable of predicting which IUD user colonized with Actinomyces may subsequently develop severe pelvic infection. All IUD users should have an annual gynecological follow-up, with specific attention paid to inflammatory symptoms. In addition, it is recommended that IUDs be replaced every 5 years. IUD is often a concomitant factor; hence its extraction is a relevant part of management as superficial endometrial infection may be shed with the menstrual period.

In pelvic inflammatory disease, it is convenient to remove the IUD and treat for 2-6 weeks with wide spectrum antibiotic coverage. In suspected Actinomyces suppurative genital and pelvic inflammatory disease, intravenous and subsequent long-term oral penicillin therapy produces rapid clinical improvement and results in a significant decrease in the size of the pelvic mass. Antibiotics and nonsteroidal anti-inflammatory drugs should be initiated as soon as possible. High dose penicillin-based therapy should be used for long periods, and drainage or resection of purulent masses may increase antibiotic efficacy. In penicillin allergic patients, clindamycin, erythromycin, or tetracycline may be appropriate. In some cases, treatment should be given continuously or intermittently for several months associated with nonsteroidal anti-inflammatory drugs.

The inability to preoperatively diagnose the acute disease and the lack of response to antibiotics (even after IUD extraction) may lead to high rates of surgical interventions not devoid of risk and complications. This needs to be taken in to account if one considers the fact that many of these women are under 40 years and may desire to subsequently have children. Percutaneous or vaginal aspiration or drainage under ultrasound or radiologic control may be acceptable and less morbid alternatives than surgery. ${ }^{23,24}$ Therefore, early precise diagnosis and nonaggressive treatment modalities are mandatory to preserve health and reproductive capability. When tubal-ovarian chronic masses are the cause of recurrences, surgery should be necessary after antibiotic therapy. Fertility preservation may be compromised in chronic purulent processes.

Asymptomatic IUD using women with Actinomyces-like micro-organisms on the PAP smear, continue IUD use without further consideration. Despite this, women should receive information regarding the theoretic potential risk of future actinomycosis and the limited value of the finding due to their commensal nature.

\section{Disclosures}

The authors report no conflicts of interest in this work.

\section{References}

1. Bowden GHW. Actinomyces, Propionibacterium propionicus, and Streptomyces. Medical Microbiology, University of Texas at Galveston. http://www.utmb.edu/gsbs/microbook/ch034.htm. Accessed 2010 February 5.

2. Apothélez C, Regamey C. Disseminated infection due to Actinomyces meyeri. Case report and review. 1996;4:621-625.

3. Cancer or actinomycosis? Chronicle. 1894 November 30;38(12120):2. http://paperspast.natlib.govt.nz/cgi-bin/paperspast?a=d\&d=WC189411 30.2.25. Accessed 2010 February 5.

4. Westhoff C. IUDs and colonization or infection with Actinomyces. Contraception. 2007;75 6 Suppl:S48-50.

5. Woman who visited doctors 1,000 times over 20 years. Daily Mail, 2008 October 24. http://www.dailymail.co.uk/health/article-1080293/ Woman-visited-doctors-1-000-times-20-years-finally-told-whatswrong-incurable.html. Accessed 2010 February 5.

6. Persson E, Holmberg K, Dahlgren S, Nilsson L. Actinomyces israelii in the genital tract of women with and without intrauterine contraceptive devices. Acta Obstet Gynecol Scand. 1983;62:563-568.

7. Justesen T, Nielsen OH, Jacobsen IE, Lave J, Rasmussen SN. The normal cultivable microflora in upper jejunal fluid in healthy adults. Scand $J$ Gastroenterol. 1984;19:279-282.

8. Evans DT. Actinomyces israelii in the female genital tract: a review. Genitourin Med. 1993;69:54-59.

9. Fiorino AS: Intrauterine contraceptive device-associated actinomycotic abscess and Actinomyces detection on cervical smear. Obstet Gynecol. 1996;87:142-149.

10. Wai CY, Nihira MA, Drewes PG, Chang JS, Siddiqui MT, Hemsell DL. Actinomyces associated with persistent vaginal granulation tissue. Infect Dis Obstet Gynecol. 2005;13:53-55.

11. Toh ST, Yuen HW, Goh YH. Actinomycetes colonization of tonsils: a comparative study between patients with and without recurrent tonsillitis. J Laryngol Otol. 2007;121:775-778.

12. Martinez-Girón R, Torre-Bayón C, Tamargo-Peláez ML, LópezCabanilles MD, Ribas-Barceló A. Actinomyces-like organisms on a pap smear. Acta Cytol. 2009;53:483-485.

13. Women told to replace IUDs every two years. Free Lance Star, 1981 March 3. http://news.google.es/newspapers?id=k1MQAAAAIBAJ\&s $\mathrm{jid}=\mathrm{h} 4 \mathrm{sDAAAAIBAJ} \& \mathrm{pg}=5219,174604 \& \mathrm{dq}=$ actinomycosis + woman\&hl=en. Accessed 2010 February 5.

14. Smego R, Foglia G. Actinomycosis. Clin Infect Dis. 1998; 26:1255-1263.

15. Simms I, Stephenson JM. Pelvic inflammatory disease epidemiology: what do we know and what do we need to know? Sex Transm Infect. 2000;76:80-87.

16. Chatwani A, Amin-Hanjani S: Incidence of actinomycosis associated with intrauterine devices. J Reprod Med. 1994;39:585-587. 
17. Merki-Feld GS, Lebeda E, Hogg B, Keller PJ. The incidence of actinomyces-like organisms in Papanicolaou-stained smears of copper- and levonorgestrel-releasing intrauterine devices. Contraception. 2000;61:365-368.

18. Valicenti JF, Pappas AA, Graber CD, Williamson HO, Willis NF. Detection and prevalence of IUD-associated actinomyces colonisation and related morbidity. JAMA. 1982;247:1149-1152.

19. Bhagavan BS, Gupta PK. Genital actinomycosis and intrauterine contraceptive devices. Cytopathologic diagnosis and clinical significance. Hum Pathol. 1978;9:567-578.

20. Dybdahl H, Hastrup J, Banndrup U. The clinical significance of actinomyces colonisation as seen in cervical smears. Acta Cytol. 1991;35:142-143.
21. López-Olmos J, Gasull J, Vivar B. Actinomyces and mixed infections in cervicovaginal smears in intrauterine device wearers. Clín Investig Ginecol Obstet. 2010; in press. doi:10.1016/j.gine.2009.05.004.

22. Antonelli D, Kustrup JF Jr. Large bowel obstruction due to intrauterine device: associated pelvic inflammatory disease. Am Surg. 1999;65:1165-1166.

23. Lee YC, Min D, Holcomb K, Buhl A, DiMaio T, Abulafia O. Computed tomography guided core needle biopsy diagnosis of pelvic actinomycosis. Gynecol Oncol. 2000;79:318-323.

24. Gjelland K, Ekerhovd E, Granberg S. Transvaginal ultrasound-guided aspiration for treatment of tubo-ovarian abscess: a study of 302 cases. Am J Obstet Gynecol. 2005;193:1323-1330.
Open Access Journal of Contraception

\section{Publish your work in this journal}

Open Access Journal of Contraception is an international, peerreviewed, open access, online journal, publishing original research, reports, reviews and commentaries on all areas of contraception. In addition to clinical research, demographics and health-related aspects, the journal welcomes new findings in animal and preclinical studies

\section{Dovepress}

relating to understanding the biological mechanisms and practical development of new contraceptive agents. The manuscript management system is completely online and includes a very quick and fair peer-review system. Visit http://www.dovepress.com/testimonials.php to read real quotes from published authors. 